Original Paper http://ajol.info/index.php/ijbcs http://indexmedicus.afro.who.int

\title{
Etude des paramètres opératoires d'électrocoagulation pour le traitement d'un effluent de textile : exemple du bleu de méthylène
}

\author{
Yibor Fabrice Roland BAKO ${ }^{1}$, Inoussa ZONGO ${ }^{2}$, Yssouf KARANGA ${ }^{1,3}$, \\ Issa TAPSOBA $^{1 *}$, Issoufou SAWADOGO ${ }^{1}$ et Barthélemy BAGA ${ }^{1}$
}

${ }^{1}$ Laboratoire de Chimie Analytique, Environnementale et Bioorganique (LCAEBiO), Département de Chimie, UFR/SEA, Université Joseph Ki-Zerbo, 03 BP 7021 Ouagadougou 03, Burkina Faso.

${ }^{2}$ Laboratoire des technologies de l'environnement et des produits naturels, Institut de Recherche en Sciences appliquées et technologies (IRSAT), Centre National de la Recherche Scientifique et Technologique (CNRST) 03 BP 7047 Ouagadougou 03, Burkina Faso.

${ }^{3}$ Laboratoire de Chimie des Matériaux et de l'Environnement, (LCME), Université Norbert ZONGO, Koudougou, Burkina Faso.

*Auteur correspondant ; E-mail : issa.tapsoba@gmail.com ; Tél. (00226) 706255 36/79 179935

\author{
Received: 06-08-2020 \\ Accepted: 19-03-2021 \\ Published: 30-04-2021
}

\section{RESUME}

Les effluents industriels issus des activités textiles présentent une grande charge polluante difficilement biodégradable et qui a des impacts non négligeables sur l'environnement et l'Homme. Leur décontamination par les procédés conventionnels biologiques ou physiques est souvent inefficace et nécessite par conséquent le recours à des procédés d'oxydation avancée dont l'électrocoagulation. Dans le présent travail, nous avons étudié l'élimination par électrocoagulation du bleu de méthylène, modèle de colorant synthétique textile, en utilisant des électrodes de fer et d'aluminium. L'étude des paramètres pouvant influencer l'élimination par électrocoagulation du bleu de méthylène tels que le $\mathrm{pH}$, la durée de l'électrolyse, la densité de courant et la nature de l'anode a montré que les meilleurs traitements sont obtenus avec un effluent de pH égal à 7 pendant 4 heures d'électrolyse à une densité de courant de $3,75 \mathrm{~A} / \mathrm{cm}^{2}$ en utilisant l'électrode de fer. Dans ces conditions, le pourcentage d'élimination du bleu de méthylène dans les eaux atteint 80,1\%. Des électrodes en fer seraient donc plus intéressantes pour l'élimination du bleu de méthylène, comparativement à des électrodes en aluminium. (C) 2021 International Formulae Group. All rights reserved.

Mots clés : Electrocoagulation, fer, aluminium, effluents industriels, bleu de méthylène.

\section{Study of operator parameters in electrocoagulation for the treatment of a synthetic textile effluent: example of methylene blue}

\begin{abstract}
Industrial effluents from textile activities have a large polluting load that is difficult to biodegrade and which has significant impacts on the environment and on humans. Their decontamination by conventional biological or physical processes is often ineffective and therefore requires the use of advanced oxidation processes including electrocoagulation. In the present work, we investigated the electrocoagulation removal of methylene blue, a synthetic textile dye model, using iron and aluminum electrodes. The study of the parameters
\end{abstract}


of EC which can influence the elimination of methylene blue in wastewater, such as the $\mathrm{pH}$, the duration of the electrolysis, the current density and the nature of the anode showed that the best treatment are obtained with a $\mathrm{pH}$ effluent equal to 7, for 4 hours of electrolysis with an iron electrode at a current density of $3.75 \mathrm{~A} / \mathrm{cm}^{2}$. Under these conditions, the percentage of elimination of methylene blue in water reaches $80.1 \%$. Iron electrodes would therefore be more advantageous for the removal of methylene blue, compared to aluminum electrodes. (C) 2021 International Formulae Group. All rights reserved.

Keywords: Electrocoagulation, iron, aluminum, industrial effluents, methylene blue.

\section{INTRODUCTION}

Les besoins en eau augmentent régulièrement avec l'accroissement démographique et l'industrialisation de nombreux pays émergents. À l'échelle mondiale, la protection des ressources en eau est une préoccupation importante. L'eau, placée au cœur des objectifs du développement durable (objectif 6) visant à garantir l'accès de tous à l'eau, l'assainissement et assurer une gestion durable des ressources en eau, est un élément crucial pour la réalisation de tous les autres objectifs, notamment la promotion d'un travail décent pour tous, thème sur lequel est axée l'édition 2016 du rapport mondial sur la mise en valeur des ressources en eau (PMERE, 2016). Plus de 700 millions de personnes n'ont toujours pas accès à l'eau potable toujours selon l'édition 2016 du rapport mondial des Nations Unies sur la mise en valeur des ressources en eau (PMERE, 2016). A l'occasion de la Journée Mondiale de l'Eau du mardi 22 mars 2016, «Solidarité Internationale » rappelle que 2,6 millions de personnes (dont 1,8 million d'enfants) meurent encore chaque année de maladies liées à l'eau insalubre, soit une personne toutes les 10 secondes (PMERE, 2016). Selon l'ONG WaterAid, 3,4 millions de personnes au Burkina-Faso n'ont pas accès à l'eau potable (Maba, 2014). Le principal problème de l'accès à l'eau potable est dû à une mauvaise répartition dans le monde mais aussi à une pollution continue des ressources en eau par l'agriculture, les rejets urbains et l'industrie (Khemis et al., 2006).

Les industries textiles sont de grands consommateurs d'eau. Pour teindre 30 milliards de kilogrammes de tissus par an, il faut consommer 4 milliards de tonnes d'eau par an (Maba, 2014). Cela engendre des rejets liquides très importants et chargés de colorants dans l'environnement, bien qu'il soit établi que bon nombre des colorants utilisés dans l'industrie textile sont toxiques, mutagènes et génotoxiques pour la faune et les humains (Mahmood et al., 2016). De plus, en raison de leurs photo et thermo stabilités élevées, ces composés peuvent persister longtemps dans l'environnement (Bhatia et al., 2017). Au regard de tous ces effets néfastes, un traitement de ces eaux usées s'impose avant leur rejet dans le milieu naturel.

Pour ce faire, plusieurs procédés de traitement sont mis en œuvre (Golder et al., 2005 ; Guo et al., 2013 ; Zodi et al., 2013 ; De Carvalho et al., 2015 ; Khemila et al., 2018 ; Collivignarelli et al., 2019 ; Pavithra et al., 2019 ; Wong et al., 2019 ; Zazou et al., 2019). La coagulation chimique et les traitements biologiques sont des techniques utilisées particulièrement pour le traitement de divers types d'effluents mais ils ont pour inconvénients de provoquer une acidification de l'eau traitée, une production d'importantes quantités de boues et ne sont pas toujours efficaces pour les effluents textiles. Pour relever ce défi, l'électrocoagulation a été développée. Le présent travail est consacré à l'étude du traitement d'un effluent textile par électrocoagulation.

Le procédé d'électrocoagulation crée, au sein de l'eau que l'on souhaite épurer, des flocs d'hydroxydes métalliques par électrodissolution d'anodes solubles (généralement, le fer et l'aluminium). Selon la valeur du $\mathrm{pH}$, l'aluminium et le fer s'oxydent après le passage d'un courant pour former des complexes de type $\mathrm{Al}_{2}(\mathrm{OH})_{5}{ }^{+}, \mathrm{Al}_{2}(\mathrm{OH})^{2} 4^{+}$, ou des hydroxydes peu solubles $\mathrm{Al}(\mathrm{OH})_{3}$, $\mathrm{Fe}(\mathrm{OH})_{2}$ ou $\mathrm{Fe}(\mathrm{OH})_{3}$. Ce sont ces dernières formes qui jouent le rôle de coagulant (Zongo, 2009). Ainsi, des agrégats de particules sont alors formés et vont décanter pour laisser place 
à une eau claire et "épurée". Le champ électrique, au passage du courant dans la cellule électrolytique entraîne la migration des particules colloïdales vers l'anode, ce qui a pour effet d'accroître leur probabilité de rencontre, donc de favoriser la coagulationfloculation. L'électrolyse de l'eau entraîne aussi la formation de petites bulles d'oxygène et d'hydrogène respectivement à l'anode et à la cathode. Cette réaction secondaire peut provoquer la flottation des matières floculées. De nombreux travaux ont été réalisés à travers le monde sur le traitement d'effluent par électrocoagulation, mais la plupart ne concernait que des effluents issus des industries textiles et pas le traitement du bleu de méthylène qui est pourtant le colorant le plus utilisé par les artisans de la teinture des pagnes traditionnels au Burkina Faso. L'objectif de ce travail est d'étudier les paramètres opératoires pour l'élimination du bleu de méthylène utilisé par les teinturiers des pagnes traditionnels au Burkina Faso par électrocoagulation avec une mention sur la comparaison de l'utilisation des électrodes de fer et celles d'aluminium.

\section{MATERIEL ET METHODES}

\section{Caractéristiques de l'effluent (bleu de méthylène) et réactifs utilisés}

$\mathrm{Au}$ cours de cette étude, le bleu de méthylène a été utilisé comme molécule modèle. L'effluent utilisé a été préparé au laboratoire par dissolution du bleu de méthylène dans de l'eau distillée. Il est d'origine FLUKA et est utilisé sans purification préalable.

Le bleu de méthylène est un composé cationique de caractère oxydant (Abbaz et al., 2014). Sa toxicité et son caractère non biodégradable est un obstacle à son utilisation ou au recyclage de l'eau par les procédés classiques. Ses principales caractéristiques sont présentées dans le Tableau 1.

Le bleu de méthylène est un colorant azinique (thiazine), cationique aussi appelé chlorure de méthylthioninium, qui est un composé organique dont le nom en nomenclature systématique est le 3,7-bis(dimethylamino)phenazathionium. Il est couramment utilisé comme modèle de contaminant organique en raison de sa structure moléculaire stable. Il se présente sous forme d'une poudre cristalline d'un bleu foncé et est un dérivé azoté (Figure 1). Dans la réaction de réduction, le bleu de méthylène se transforme en leuco méthylène incolore, due à la perte du doublet libre de l'azote et introduction d'un atome d'hydrogène (Figure 2).

La solution mère utilisée, de concentration $1000 \mathrm{mg} / \mathrm{l}$ est obtenue en dissolvant 0,1 gramme de bleu de méthylène dans une fiole de $100 \mathrm{ml}$ et en complétant avec de l'eau distillée milli Q $18 \mathrm{M} \Omega$. Pour établir la courbe d'étalonnage, différentes solutions ont été préparées à partir de la solution mère par dilution, leurs concentrations variant de 1 à 10 $\mathrm{mg} / \mathrm{l}$.

L'eau distillée a été préparée au laboratoire sur distillateur type GFL 2001/4 et purifié à travers une membrane type SCIAQUA ULTRA + pour avoir de l'eau distillée milli-Q de résistance $18 \mathrm{M} \Omega$. Le $\mathrm{pH}$ de l'effluent simulé a été ajusté par une solution aqueuse d'hydroxyde de sodium $(\mathrm{NaOH})$ d'origine Sigma à l'aide d'un $\mathrm{pH}$-mètre de type Metrohm 691. Le dihydrogénophosphate $\left(\mathrm{K}_{2} \mathrm{HPO}_{4}\right)$, l'hydrogénophosphate $\left(\mathrm{KH}_{2} \mathrm{PO}_{4}\right)$, le chlorure de sodium $(\mathrm{NaCl})$, la soude caustique $(\mathrm{NaOH})$ et l'acide sulfurique $\left(\mathrm{H}_{2} \mathrm{SO}_{4}\right)$ sont d'origine FLUKA et ont été utilisé comme tels.

Pour la préparation de la solution tampon, on réalise un mélange équimolaire de dihydrogénophosphate $\left(\mathrm{K}_{2} \mathrm{HPO}_{4}\right)$ et d'hydrogénophosphate $\left(\mathrm{KH}_{2} \mathrm{PO}_{4}\right)$ à $0,1 \mathrm{M}$. On introduit dans deux fioles jaugées de $500 \mathrm{ml}$ contenant au préalable un peu d'eau distillée milli-Q, $17,2 \mathrm{~g}$ de $\mathrm{K}_{2} \mathrm{HPO}_{4}$ et $13,61 \mathrm{~g}$ de $\mathrm{KH}_{2} \mathrm{PO}_{4}$. On mélange les deux solutions et on homogénéise. On obtient ainsi 1 litre de tampon phosphate à $\mathrm{pH}$ sensiblement égal à 7 . La soude caustique et l'acide sulfurique ont été utilisés pour fixer le $\mathrm{pH}$ de la solution.

\section{Description du montage expérimental}

Le dispositif expérimental a été monté au laboratoire et est constitué de : 
- un bécher en pyrex de $120 \mathrm{ml}$ qui joue le rôle du réacteur électrochimique;

- un potentiostat de marque Digi-Ivy modèle DY2322 servant de générateur de courant électrique piloté par un logiciel;

- des électrodes en fer ou en aluminium : les électrodes sont placées parallèlement l'une par rapport à l'autre. Les dimensions des électrodes sont $40 \times 20 \times 1 \mathrm{~mm}$ et la surface des électrodes est de $8 \mathrm{~cm}^{2}$.

- un fil de platine comme contre électrode ;

- une électrode de graphite pour la mesure électrochimique ;

- une électrode au calomel saturé (ECS) comme électrode de référence

- Un agitateur magnétique pour rendre l'effluent homogène dans le bécher.

Le schéma du dispositif est représenté par la Figure 3.

\section{Méthode d'analyse du bleu de méthylène}

L'élimination du bleu de méthylène en utilisant le procédé d'électrocoagulation a été suivie par la méthode d'analyse électrochimique en suivant son pic de réduction en fonction du potentiel. La méthode basée sur le comportement électrochimique du bleu de méthylène a été développée pour analyser l'évolution du bleu de méthylène dans l'effluent simulé.

Avant de procéder à l'élimination du bleu de méthylène par électrocoagulation, la réponse électrochimique du bleu de méthylène a été enregistrée en voltammétrie à signaux carrés sur une électrode de graphite dans un tampon phosphate $\mathrm{pH}=7$. Les paramètres de mesure de la réponse électrochimique notamment la pulsation, la fréquence et l'amplitude ont ainsi été optimisés.

\section{Traitement de l'effluent de bleu de méthylène}

Le bécher est rempli avec $100 \mathrm{~mL}$ de l'effluent synthétique à traiter de concentration initiale $10 \mathrm{mg} / \mathrm{L}$ et ceci dans les conditions opératoires choisies. La conductivité de la solution est ajustée avec du chlorure de sodium $(\mathrm{NaCl})$. Lorsque les électrodes de fer ou d'aluminium sont utilisées, des couches d'oxydes de $\mathrm{Al}_{2} \mathrm{O}_{3}$ et $\mathrm{Fe}_{3} \mathrm{O}_{4}$ protectrices peuvent être formées lorsqu'elles sont exposées à l'air. Ces deux formes sont aussi les oxydes de protection lorsque les métaux se trouvent plongés dans l'effluent assez longtemps sans réactions électrochimiques. Néanmoins, en présence d'ions chlorures, cette relative passivation n'a plus lieu et la corrosion des électrodes se produit. Le choix du chlorure de sodium découle du fait que les ions chlorures réduisent de manière significative ces effets indésirables (Guo et al., 2013; Pavithra et al., 2019). Le chlorure de sodium, utilisé en tant qu'électrolyte support, est nécessaire au bon déroulement d'une réaction d'électrocoagulation, en empêchant la formation de dépôts sur la surface de l'anode et en accélérant la dissolution de celle-ci et donc la cinétique de réaction (Bensaid, 2009).

Les différentes valeurs du $\mathrm{pH}$ sont mesurées avec un pH-mètre de type Metrohm 691 ; elles sont obtenues par ajustement de la solution à l'aide d'une solution de soude $(\mathrm{NaOH})$ ou de l'acide sulfurique $\left(\mathrm{H}_{2} \mathrm{SO}_{4}\right)$. Trois électrodes sont placées dans la cellule électrochimique : une électrode de travail en fer, une ECS comme électrode de référence et une contre électrode en platine. La distance inter-électrode est de $2 \mathrm{~cm}$. Après chaque traitement, les électrodes sont frottées avec du papier abrasif et rincés avec de l'eau distillée afin d'enlever les dépôts sur les électrodes. L'optimisation des conditions opératoires a consisté à varier alternativement chacun des paramètres suivants, l'un indépendamment des autres : le $\mathrm{pH}$ de la solution, le temps de traitement, la densité de courant et la nature de l'anode.

Après chaque électrolyse, l'effluent traité est filtré puis analysé par voie électrochimique afin de mesurer le courant correspondant au bleu de méthylène résiduel. Pour chaque expérience, le taux d'abattement ou d'élimination du bleu de méthylène est estimé à partir de la formule suivante :

$$
\mathrm{E}(\%)=\frac{\mathrm{C}_{0}-\mathrm{C}_{\mathrm{r}}}{\mathrm{C}_{0}} \times 100
$$

$\mathrm{C}_{0}$ : Concentration initiale de bleu de méthylène en $\mathrm{mg} / \mathrm{l}$.

$\mathrm{C}_{\mathrm{r}}$ : Concentration résiduelle de bleu de méthylène en $\mathrm{mg} / \mathrm{l}$.

E (\%) : Taux d'élimination en pourcentage. 
Tableau 1 : Principales caractéristiques du bleu de méthylène (Bouaziz Karime, 2014).

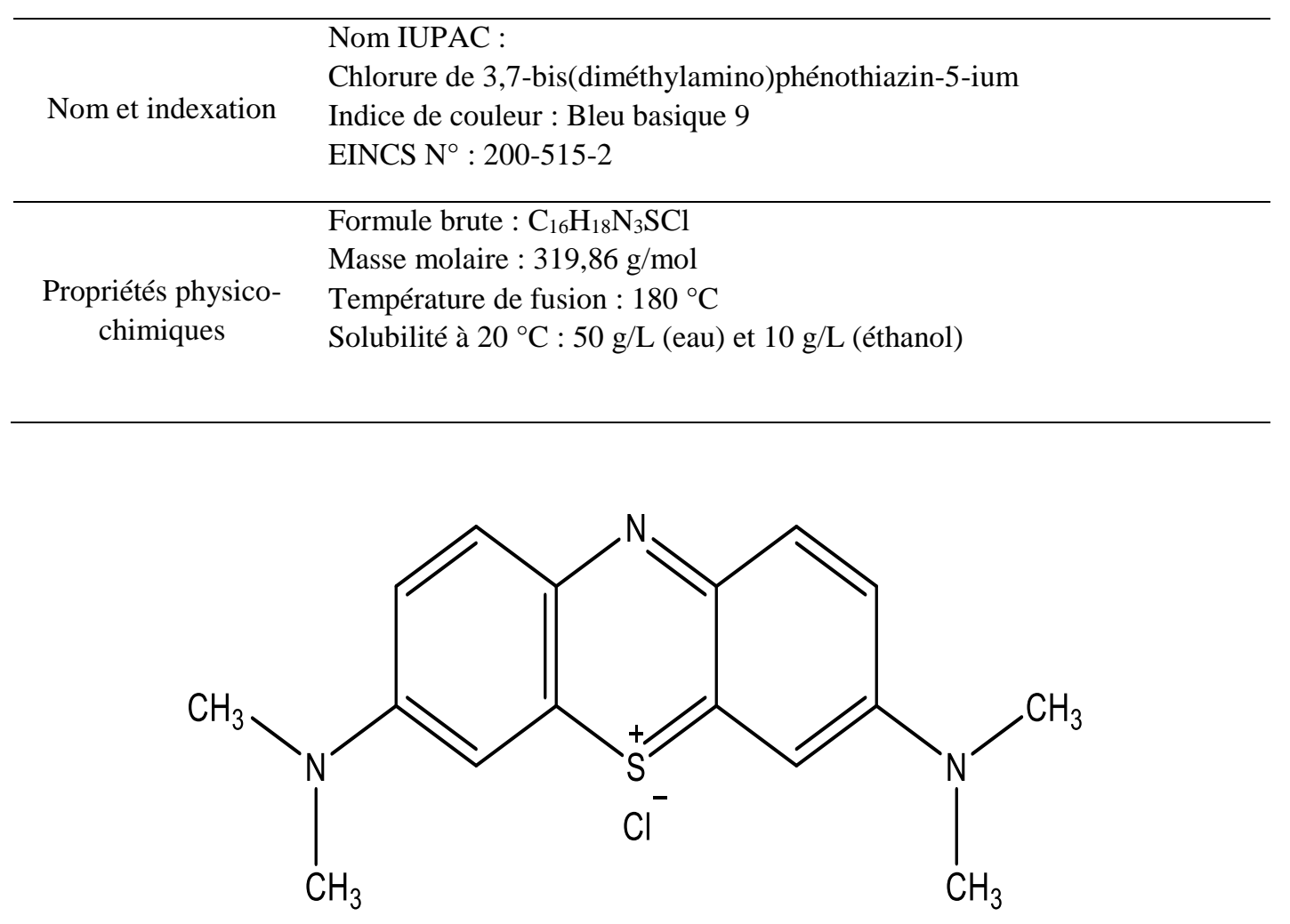

Figure 1 : Structure chimique du bleu de méthylène (Maba, 2014).<smiles>CN(C)c1ccc2c(c1)Sc1cc(N(C)C)ccc1N2</smiles>

Figure 2 : Forme incolore du bleu de méthylène. 


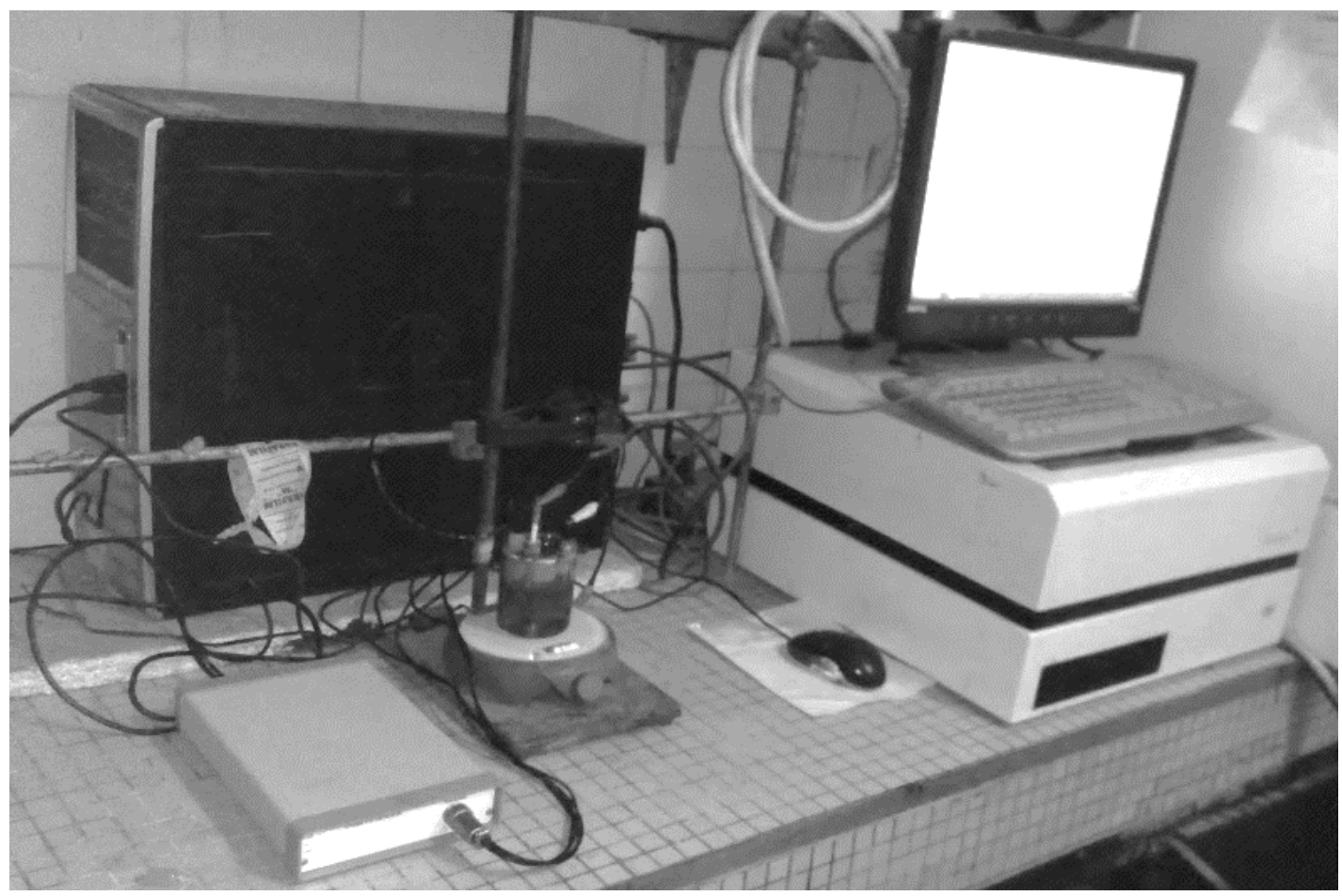

Figure 3 : Photo du dispositif expérimental.

\section{RESULTATS}

\section{Réponse électrochimique du bleu de} méthylène

L'enregistrement de la réponse électrochimique du bleu de méthylène en voltammétrie à signaux carrés a donné le voltammogramme de la Figure 4. Ce voltammogramme présente un pic au potentiel de $-0,42 \mathrm{~V} / \mathrm{ECS}$ qui correspond à la réduction du bleu de méthylène (Bouaziz Karime, 2014). L'étude de l'effet de la concentration du bleu de méthylène sur son pic de réduction a donné les voltammogrammes présentés sur la Figure 5. Il ressort de cette figure que le courant de pic de réduction augmente avec la concentration du bleu de méthylène. Par ailleurs, le graphique du courant de pic cathodique en fonction de la concentration (Figure 6) montre que cette variation du courant de pic est linéaire dans deux plages de concentrations. Ainsi, pour des concentrations supérieures à $4 \mathrm{mg} / \mathrm{l}$, le courant du pic est donné par la relation $I p=$ 13,087C - 49,621, $\mathrm{I}_{\mathrm{p}}$ désignant l'intensité du pic et $\mathrm{C}$ la concentration du bleu de méthylène.
Dans le cas des concentrations inferieures à 4 $\mathrm{mg} / \mathrm{l}$, nous avons plutôt la relation $I_{p}=0,7 \mathrm{C}-$ 0,133 .

\section{Influence de la nature de l'électrode à différents pH}

La Figure 7 confronte les résultats de l'élimination du bleu de méthylène par des électrodes de fer et d'aluminium de mêmes dimensions. Un examen approfondi de cette figure montre que le fer et l'aluminium sont efficaces pour l'élimination du bleu de méthylène. On observe aussi que les taux d'élimination sont meilleurs avec le fer en milieu acide tandis qu'en milieu basique, les meilleurs taux d'élimination sont obtenus avec l'aluminium. De plus, on note que l'élimination est maximale à $\mathrm{pH} 7$ en milieu acide et à pH 11 en milieu basique.

\section{Influence de la densité de courant}

La Figure 8 illustre l'effet de la densité $\mathrm{du}$ courant sur l'élimination du bleu de méthylène en utilisant une électrode de fer. Il 
ressort de cette figure que le taux d'élimination augmente avec la densité de courant avec un meilleur taux obtenu de $52,4 \%$ pour une densité de courant égale à $3,75 \mathrm{~A} / \mathrm{cm}^{2}$.

\section{Influence de la durée de l'électrolyse}

La Figure 9 présente l'effet du temps de traitement de l'effluent synthétique sur le taux d'élimination du bleu de méthylène avec une électrode de fer. D'après les données de cette figure, le taux d'élimination varie avec le temps d'électrolyse. On note que ce taux a un maximum de $80,1 \%$ pour une durée de traitement de $4 \mathrm{~h}$.

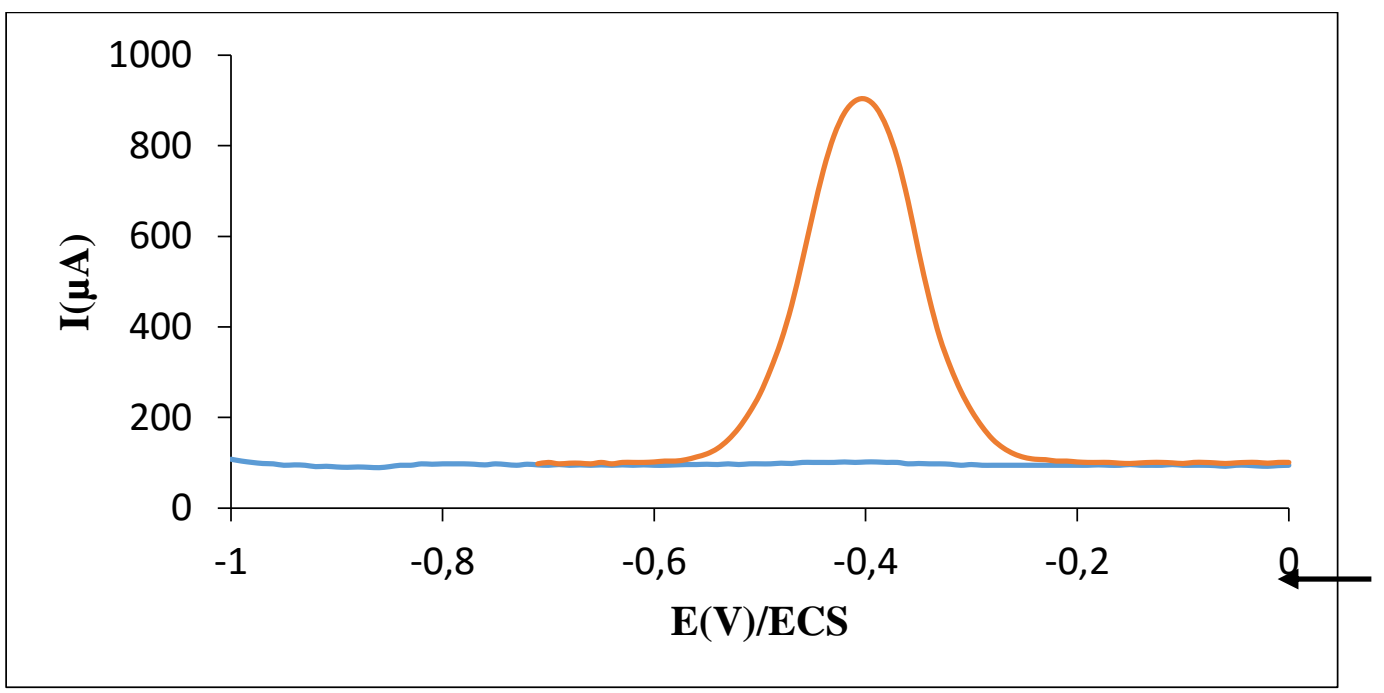

Figure 4 : Voltamogramme à signaux carrés du bleu de méthylène (concentration $\mathrm{C}=10 \mathrm{mg} / \mathrm{L}$ ) dans le tampon phosphate $\mathrm{pH}=7$ sur une électrode de carbone en graphite $(\phi=1 \mathrm{~mm})$, électrode de référence : ECS ; Fréquence $10 \mathrm{~Hz}$; Amplitude $50 \mathrm{mV}$; Pulsation $10 \mathrm{mV}$.

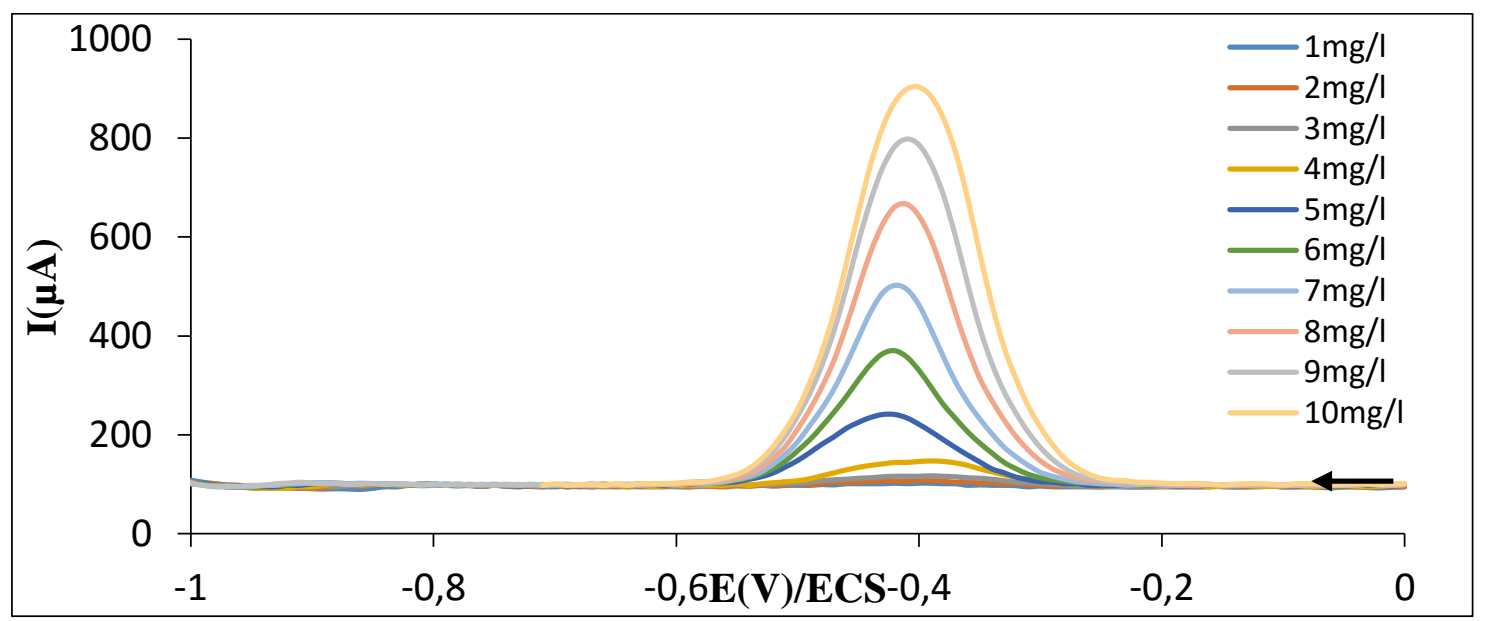

Figure 5 : Voltamogrammes à signaux carrés du bleu de méthylène en fonction de la concentration dans le tampon phosphate $\mathrm{pH}=7$ sur une électrode de carbone en graphite $(\phi=1 \mathrm{~mm})$; Electrode de référence ECS ; Fréquence $10 \mathrm{~Hz}$; Amplitude $50 \mathrm{mV}$, Pulsation $10 \mathrm{mV}$. 


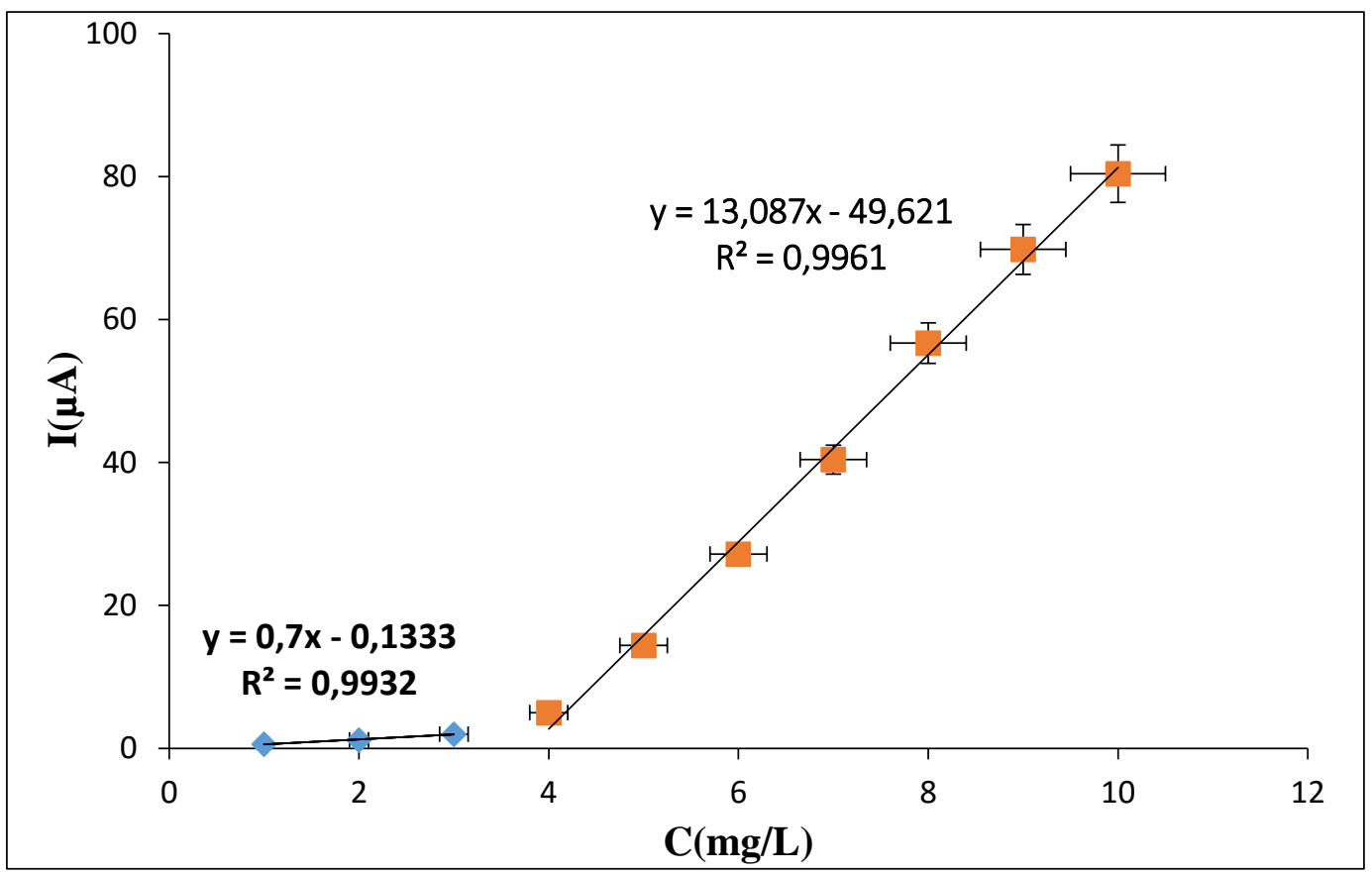

Figure 6 : Courbe d'étalonnage du bleu de méthylène.

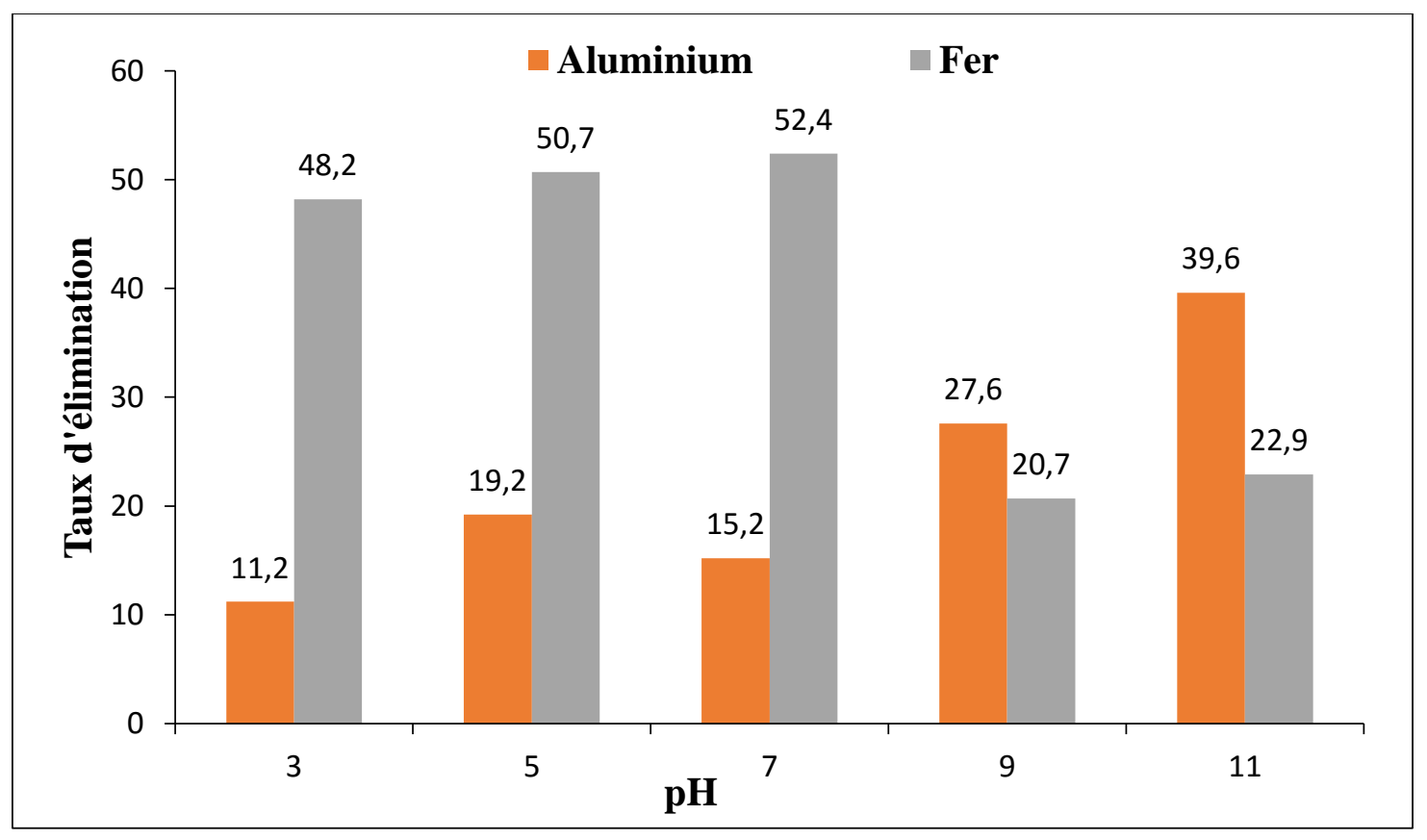

Figure 7 : Influence de la nature de l'anode. Paramètres : densité de courant $=3,75 \mathrm{~A} / \mathrm{cm}^{2}$; temps d'électrolyse $=1 \mathrm{~h}$; anodes : lames de fer et d'aluminium. 


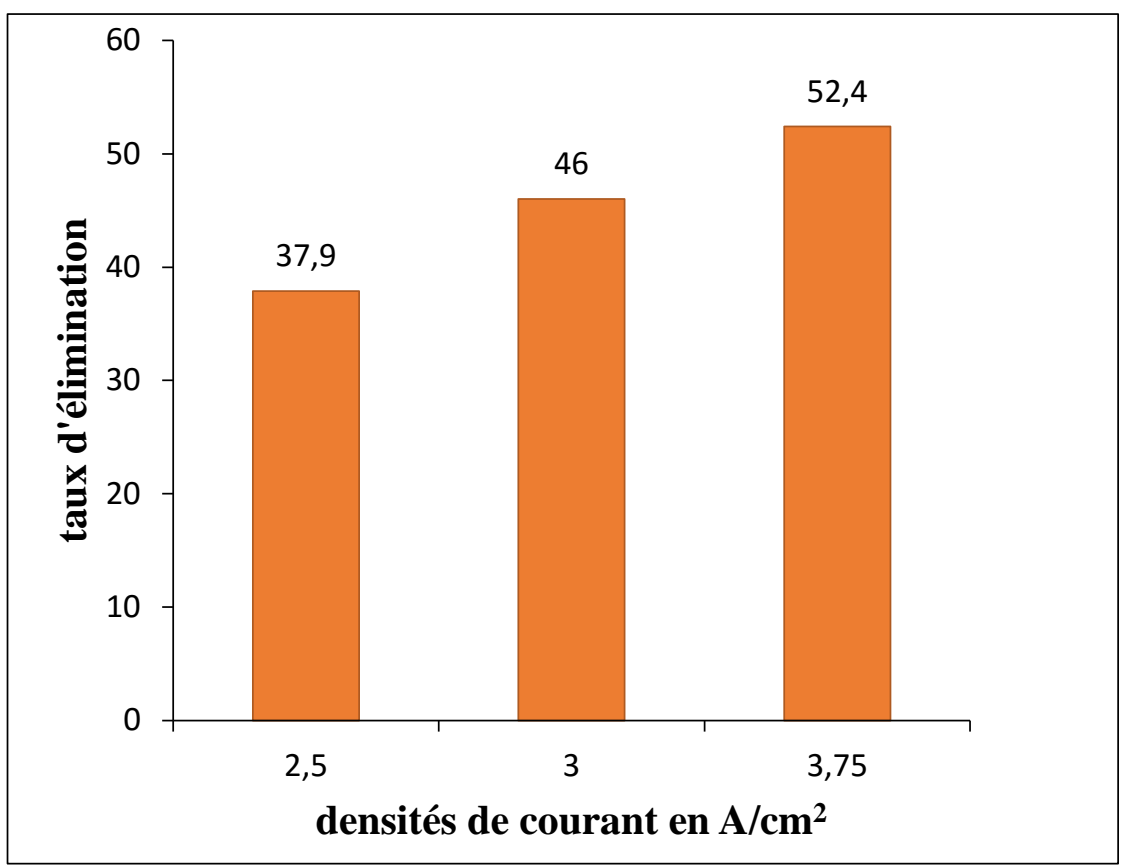

Figure 8 : Evolution du taux d'élimination du bleu de méthylène en fonction de la densité de courant. Paramètres : $\mathrm{pH}=7$; temps d'électrolyse $=1 \mathrm{~h}$; anode : lame de fer.

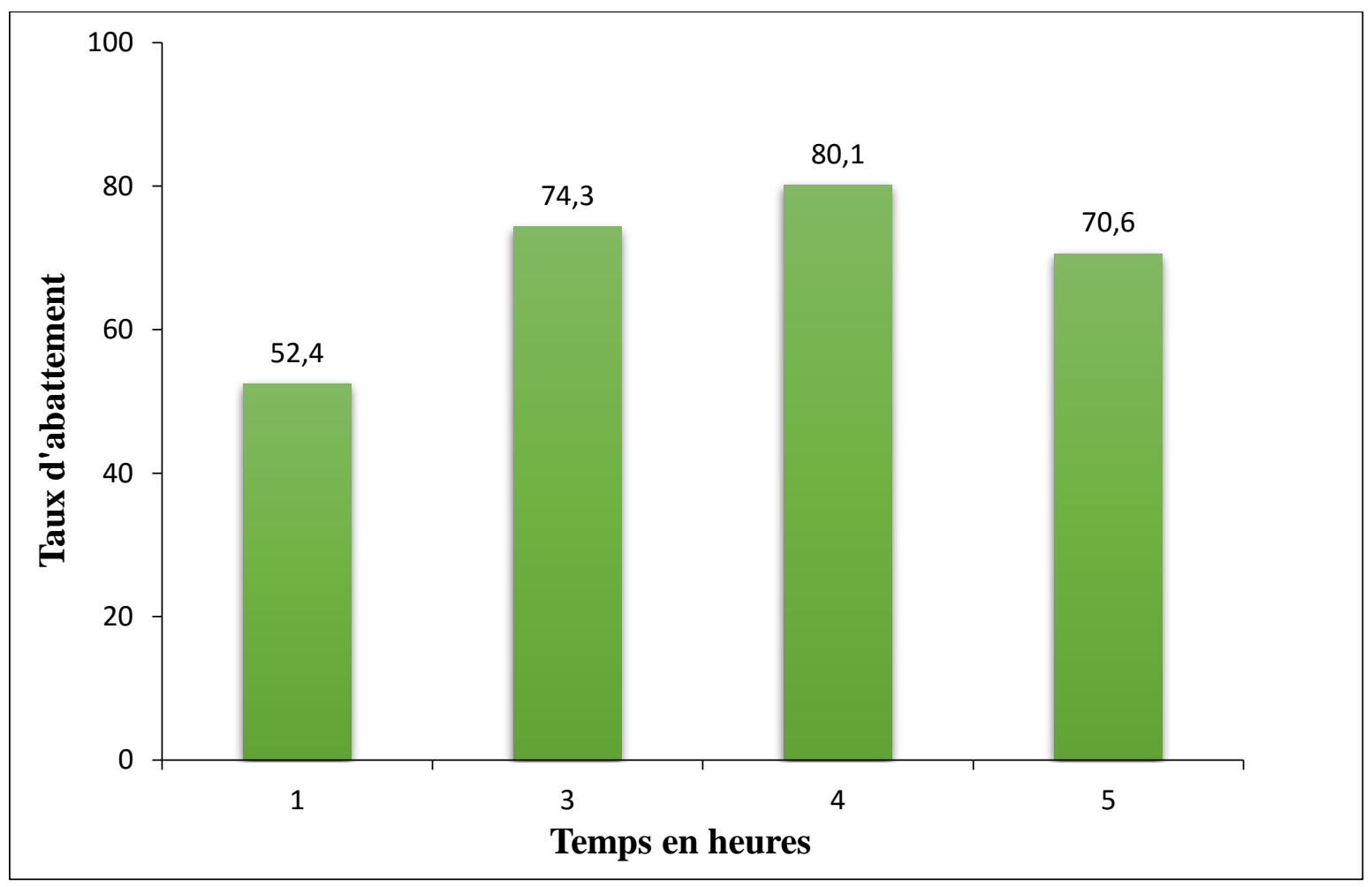

Figure 9 : Evolution du taux d'élimination du bleu de méthylène en fonction du temps. Paramètres : $\mathrm{pH}=7$; densité de courant $=3,75 \mathrm{~A} / \mathrm{cm} 2$; anode : lame de fer. 


\section{DISCUSSION}

Le but de ce travail était d'étudier les paramètres opératoires pour l'élimination du bleu de méthylène par électrocoagulation afin de déterminer les meilleures conditions, notamment la nature du matériau d'électrode (fer ou aluminium) pour une élimination optimale de ce polluant des effluents textiles.

L'influence de la nature de l'anode sur le taux d'élimination du bleu de méthylène a été étudiée en comparant l'efficacité du traitement des lames de fer avec les lames d'aluminium d'égales dimensions (Figure 7). A un $\mathrm{pH}$ proche de la neutralité, le fer permet d'atteindre son maximum d'élimination à $52,4 \%$ du bleu de méthylène alors que la lame d'aluminium présente son meilleur taux d'abattement à $39,6 \%$ au $\mathrm{pH}$ de 11 . Ces résultats sont conformes aux travaux de Lemlikchi (2012) qui ont montré que les meilleurs taux d'élimination sont obtenus avec le fer. Ils sont également comparables aux résultats obtenus par Zongo (2009) qui a obtenu $100 \%$ d'abattement avec les électrodes de fer contre $70 \%$ avec celle en aluminium sur les effluents pollués en chromes.

La faible élimination observée aux $\mathrm{pH}$ faibles pourrait s'expliquer par des réactions secondaires. En effet à ce $\mathrm{pH}$, le métal pourrait être attaqué par l'acide et conduire à une pollution métallique des eaux. Toutefois, à des valeurs de $\mathrm{pH}$ proches de la neutralité, le fer s'oxyde et les ions formés réagissent avec l'eau pour former des complexes de type $\mathrm{Fe}(\mathrm{OH})_{2}$ ou $\mathrm{Fe}(\mathrm{OH})_{3}$ (Zongo, 2009). Ce sont ces dernières formes qui jouent le rôle de coagulant. Des agrégats de particules sont alors formés et peuvent décanter pour laisser place à une eau claire.

Par ailleurs, les meilleurs taux d'élimination obtenus en milieu acide par le fer et plutôt avec l'aluminium en milieu basique sont en accord avec les données de la littérature qui montrent que l'électrode de fer est efficace pour l'élimination des composés organiques avec le procédé d'électrocoagulation dans les rejets industriels alors que l'électrode en aluminium est couramment utilisée pour l'élimination des métaux lourds (Chou et al., 2009).

De tout ce qui précède, il ressort que l'électrode de fer serait plus efficace que l'électrode d'aluminium pour l'élimination du bleu de méthylène des eaux. Ainsi, l'électrode de fer a été retenue pour la suite des expériences.

La densité de courant et la durée d'imposition du courant à l'électrode étant aussi des paramètres essentiels en électrocoagulation, leurs effets sur le taux d'élimination du bleu de méthylène ont également été investigués séparément.

Ainsi, l'étude de l'influence la densité de courant (Figure 8) a montré qu'au fur et à mesure que cette dernière augmente, le taux d'élimination du bleu de méthylène augmente. Bennajah (2007), Zongo (2009) et Lemlikchi (2012) ont fait la même constatation. En effet lorsque la densité de courant augmente, on a une forte dissolution de l'anode. Il en résulte donc une production importante de flocs qui va déstabiliser les particules de polluant. Par ailleurs le taux de production des bulles d'hydrogène augmente et leur taille diminue lorsque la densité de courant augmente (Lemlikchi, 2012). Tous ces effets sont bénéfiques pour une élimination efficace du polluant par flottation (Mollah et al., 2004). Ainsi le meilleur taux d'élimination obtenu est de $52,4 \%$ pour une densité de courant maximale égale à $3,75 \mathrm{~A} / \mathrm{cm}^{2}$.

Par ailleurs, lorsque l'on fait varier la durée de l'électrolyse de $1 \mathrm{~h}$ à $5 \mathrm{~h}$, les résultats obtenus montrent que le taux d'élimination du bleu de méthylène en fonction du temps de traitement de l'effluent. Le taux d'élimination atteint une valeur maximale de $80,1 \%$ pour un temps d'électrolyse de $4 \mathrm{~h}$. Ce résultat est comparable à la valeur rapportée $(80 \%)$ par Can et al. (2003) sur le traitement d'effluent textile par électrocoagulation avec des électrodes en aluminium et celle signalée par Kobya et al. (2003) (77\%) sur les effluents textiles traités avec des électrodes en fer et en aluminium. Par contre, ce taux est largement supérieur à celui trouvé par Muthukumar et al. 
(2007) qui est de 60\% sur les effluents acides oranges 10 en utilisant des électrodes de fer.

\section{Conclusion}

Dans ce travail nous avons étudié le traitement d'effluents textiles synthétiques par électrocoagulation en utilisant des électrodes de fer et d'aluminium et le bleu de méthylène comme molécule modèle. Ainsi, en faisant passer un courant à travers une électrode dans l'effluent, nous avons pu à travers la méthode d'électrocoagulation éliminer le bleu de méthylène avec un taux d'abattement appréciable. En optimisant le pH, le choix du meilleur matériau d'électrode, la densité de courant et la durée du traitement, on trouve à travers l'expérimentation qu'en utilisant la lame de fer, un $\mathrm{pH}$ de 7 , une durée de traitement de 4 heures et une densité de courant de 37,5 $\mathrm{A} / \mathrm{cm}^{2}$, un taux d'abattement de $80,1 \%$. Cette étude doit être complétée par la réalisation d'un pilote de traitement continu en élargissant le traitement vers des molécules plus complexes telles que les PCB, les phénols, les pesticides et tous les polluants organiques persistants. L'énergie étant un sujet de contestation du procédé d'EC, une utilisation de l'énergie solaire doit aussi être envisagée.

\section{CONFLIT D'INTERETS}

Les auteurs déclarent ne pas avoir de conflit d'intérêts.

\section{CONTRIBUTIONS DES AUTEURS}

YFRB, IZ, YK et IT ont conçu et planifié les expériences. YFRB a réalisé les expériences. IT, YK et IZ ont supervisé le travail et contribué à l'interprétation des résultats. IS et $\mathrm{BB}$ ont mis à disposition les lames de fer et d'aluminium et ont aidé à la préparation des échantillons à traiter. YFRB a rédigé le manuscrit et tous les auteurs ont des commentaires critiques pour l'amélioration du manuscrit.

\section{REMERCIEMENTS}

Les auteurs remercient le réseau ANEC/ISP et son partenaire financier
'International Science Programme (ISP)' pour le soutien financier dans le renforcement des capacités scientifiques et techniques des membres du réseau à travers la coopération Sud-Sud.

\section{REFERENCES}

Abbaz M, Aba aaki R, El haouti R, Et-taleb S, Ez-zahery M, Lhanafi S, El alem N. 2014. Elimination du bleu de méthylène dans l'eau par adsorption sur le sable titanifère (Removal of methylene blue from aqueous solution by adsorption onto the sand titaniferous). J. Mater. Environ. Sci., 5(S2): 2418-2425.

Bennajah M. 2007. Traitement des rejets industriels liquide par électrocoagulation/ électroflotation en réacteur airlift. Thèse de Doctorat, Institut National Polytechnique de Toulouse, France, p. 77.

Bensaid J. 2009. Contribution à la dépollution des eaux usées par. électrocoagulation et par adsorption sur des hydroxydes d'aluminium. Thèse de Doctorat, Université Mohamed V-ABDAL, Rabat, Maroc.

Bhatia D, Sharma NR, Singh J, Kanwar RS. 2017. Biological methods for textile dye removal from wastewater: A review. Crit. Rev. Env. Sci. Tec., 47(19): 1836-1876. DOI:

http://dx.doi.org/10.1080/10643389.2017 .1393263

Bouaziz Karime I. 2014. Traitement de solutions organiques diluées par couplage adsorption-oxydation électrochimique. Thèse de Doctorat, Université Toulouse 3 Paul Sabatier, Toulouse.

Can OT, Bayramoglu M, Kobya M. 2003. Decolorization of reactive dye solutions by electrocoagulation using aluminium electrodes. Ind. Eng. Chem. Res., 42(14): 3391-3396.

DOI: https://doi.org/10.1021/ie020951g

Chou WL, Wang CT, Huang KY. 2009. Effect of operating parameters on indium (III) ion removal by iron electrocoagulation and evaluation of specific energy 
consumption. J. Hazard. Mater., 167(13): 467-474. DOI: https://doi.org/10.1016/j.jhazmat.2009.0 1.008

Collivignarelli MC, Abbà A, Miino MC, Damiani S. 2019. Treatments for color removal from wastewater: State of the art. J. Environ. Manage., 236: 727-745. DOI: https://doi.org/10.1016/j.jenvman.2018.1 1.094

Daneshvar N, Ashassi-Sorkhabi H, Tizpar A. 2003. Decolorization of orange II by electrocoagulation method. Sep. Purif. Technol., 31(2): 153-162. DOI: https://doi.org/10.1016/S13835866(02)00178-8

De Carvalho HP, Huang J, Zhao M, Liu G, Dong L, Liu X. 2015. Improvement of Methylene Blue removal by electrocoagulation/banana peel adsorption coupling in a batch system. Alexandria Engineering Journal, 54(3): 777-786.

DOI: https://doi.org/10.1016/j.aej.2015.04.003

Golder AK, Hridaya N, Samanta AN, Ray S. 2005. Electrocoagulation of methylene blue and eosin yellowish using mild steel electrodes. J. Hazard. Mater., 127(1-3): 134-140.

DOI: https://doi.org/10.1016/j.jhazmat.2005.0 6.032 .

Guo Y, Zhu Z, Qiu Y, Zhao J. 2013. Enhanced adsorption of acid brown 14 dye on calcined $\mathrm{Mg} / \mathrm{Fe}$ layered double hydroxide with memory effect. Chem. Eng. J., 219: 69-77.

DOI: https://doi.org/10.1016/j.cej.2012.12.084

Khemila B, Merzouk B, Chouder A, Zidelkhir R, Leclerc J-P, Lapicque F. 2018. Removal of a textile dye using photovoltaic electrocoagulation. Sustain. Chem. Pharm., 7: 27-35. DOI: https://doi.org/10.1016/j.scp.2017.11.004

Khemis M, Leclerc J-P, Tanguy G, Valentin G, Lapicque F. 2006. Treatment of industrial liquid waste by electrocoagulation: experimental investigations and an overall interpretation model. Chem. Ing.
Sci., 61(11): 3602-3609. DOI: https://doi.org/10.1016/j.ces.2005.12.034 Kobya M, Can OT, Bayramoglu M. 2003. Treatment of textile wastewaters by electrocoagulation using iron and aluminium electrodes. J. Hazard. Mater., 100(1-3): $\quad 163-178 . \quad$ DOI: https://doi.org/10.1016/S03043894(03)00102-X

Lemlikchi W. 2012. Elimination de la pollution des eaux industrielles par différents procédés d'oxydation et de coprécipitation. Thèse de Doctorat, Université Mouloud Mammeri de Tizi Ouzou, Algérie.

Maba B. 2014. Dégradation des effluents textiles (cas d'un colorant synthétique : le bleu de méthylène) par procédé fenton en utilisant la latérite. Mémoire de Master, Institut International d'Ingénierie de l'Eau et de l'Environnement, Ouagadougou.

Mahmood S, Khalid A, Arshad M, Mahmood T, Crowley DE. 2016. Detoxification of azo dyes by bacterial oxidoreductase enzymes. Crit. Rev. Biotechnol., 36(4): 639-651.

DOI: https://doi.org/10.3109/07388551.2015.1 004518

Mai AT. 2004. Développement des biocapteurs électrochimiques à base de tyrosinase pour la détection des polluants organiques en phase aqueuse. Thèse de Doctorat, Université Claude Bernard Lyon I, Lyon.

Mollah MYA, Pathak SR, Patil PK, Vayuvegula M, Agrawal TS, Gomes JA, Kesmez M, Cocke DL. 2004. Treatment of orange II azo-dye by electrocoagulation (EC) technique in a continuous flow cell using sacrificial iron electrodes. J. Hazard. Mater., 106(1-3): 165-171.

DOI: https://doi.org/10.1016/j.jhazmat.2004.0 3.011

Mollah MYA, Schennach R, Parga JR, Cocke DL. 2001. Electrocoagulation (EC) Science and applications. J. Hazard. Mater., $\quad \mathbf{8 4}(1)$ : $\quad 29-41 . \quad$ DOI: 
https://doi.org/10.1016/S03043894(01)00176-5.

Muthukumar M, Karuppiah MT, Bhaskar GR. 2007. Electrochemical removal of CI Acid orange 10 from aqueous solutions. Sep. Purif. Technol., 55(2): 198-205. DOI:

https://doi.org/10.1016/j.seppur.2006.11. 014

Pavithra KG, Kumar PS, Jaikumar V, Rajan PS. 2019. Removal of colorants from wastewater: A review on sources and treatment strategies. J. Ind. Eng. Chem., 75: $1-19 . \quad$ DOI: https://doi.org/10.1016/j.jiec.2019.02.01 1

PMERE (Programme Mondial sur l'Evaluation des ressources en Eau). 2016. Rapport mondial sur la mise en valeur des ressources en eau 2016 : l'eau et l'emploi. UNESCO, Paris.

Wong JKH, Tan HK, Lau SY, Yap P-S, Danquah MK. 2019. Potential and challenges of enzyme incorporated nanotechnology in dye wastewater treatment: A review. J. Environ. Chem. Eng., 7(4): 103261. DOI: https://doi.org/10.1016/j.jece.2019.1032 61

Zazou H, Afanga H, Akhouairi S, Ouchtak H, Addi AA, Akbour RA, Douch AAJ, Elmchaouri A, Duplay J, Jada A, Hamdani M. 2019. Treatment of textile industry wastewater by electrocoagulation coupled with electrochemical advanced oxidation process. J. Water Process. Eng., 28: 214221.

DOI: https://doi.org/10.1016/j.jwpe.2019.02.0 06

Zodi S, Merzouk B, Potier O, Lapicque F, Leclerc J-P. 2013. Direct red 81 dye removal by a continuous flow electrocoagulation/flotation reactor. Sep. Purif. Technol., 108: 215-222. DOI: https://doi.org/10.1016/j.seppur.2013.01. 052

Zongo I. 2009. Etude théorique et expérimentale du procédé d'électrocoagulation : application au traitement de deux effluents textiles et d'un effluent simulé de tannerie. Thèse de Doctorat, Institut National Polytechnique de Lorraine, Nancy. 